\title{
BRILLOUIN FLOW IN A MAGNETRON DIODE AS THE KINETIC MODEL LIMIT
}

\author{
A.V. Agafonov and V.S. Voronin \\ P.N. Lebedev Physical Institute, Leninsky Prosp. 53, Moscow 117924, Russia
}

\begin{abstract}
Two classes of approaches that have received the most attention to describe the important space charge dynamics in a magnetron are Brillouin flow and the double-stream kinetic model. It is supposed that in the first case electrons move parallel to the electrode surfaces and in the second case they move in cycloidal orbits performing a single turn. Precise analysis of electron dynamics in fully selfconsistent kinetic equilibria in a smooth-bore magnetron shows that for a given external magnetic field and a voltage there exists a multiplicity of natural equilibrium states, differing as to structure of electron trajectories and emission current density. The value of emission current density differs from one to another type of equilibrium and can aspire to zero under the same condition of space charge limited flow due to a large number of revolutions of electrons around the cathode. The greater the number of revolutions the closer the main parameters of the kinetic flow to the Brillouin one. The comparison of analytical calculations and the results of computer simulation of transient processes of Brillouin flow formation from initially kinetic flow are presented.
\end{abstract}

\section{INTRODUCTION}

Despite the great number of works about magnetron operation, a detailed description of electron dynamics under strong space charge influence is complicated by the nonlinear nature of a field-particles system. In particular, there is no satisfactory solution even to the problem of electron flow formation in a magnetron with a smooth anode (a coaxial magnetron diode). In the work presented here it is shown that within the framework of accepted kinetic descriptions of a coaxial magnetron diode, multiple steady states of electron flow are possible for a given diode geometry and the same set of external parameters (applied voltage and external magnetic field). These states are distinguished by the number of electron revolutions around the cathode and the current emitted from the cathode. Direct transition from kinetic flows to Brillouin flow as a limit is shown. Numerical simulation helps to investigate the dependence of steady state properties of electron flow on its history of formation. Comparison of analytical data and results of numerical simulation is made with the purpose to analyse the conditions of applicability for existing analytical models.

\section{THEORETICAL MODELS}

Usually it is supposed for a magnetron that in an initial stage magnetically insulated axially symmetric rotating electronic flow is formed. As a rule, the description of elec- tron flow is based on two models: a hydrodynamic parapotential model, or Brillouin flow[1, 2], in which electrons rotate along circular trajectories around the cathode, and a kinetic two-flow model[3, 4], in which electrons move along cycloid trajectories, beginning and coming to an end on the cathode surface. It is necessary to emphasize that in the latter case it was always supposed that an electron makes a single revolution along a cycloid independent of the geometry of the diode (plane or cylindrical).

It is easy to show for a plane diode that at the top of a cycloidal trajectory radial velocity and electromagnetic force both equal zero. Thus it is possible to "connect" another descending (that usually is done), or ascending trajectory, then continuing them symmetrically up to the cathode, i.e. the top of trajectories in the plane diode is a point of solution branching.

But for a coaxial cylindrical diode it was shown[5] that artificial connecting of trajectories is impossible: the cylindrical metrics removes degeneration. And the structure of a kinetic flow differs in that the angular movement of electrons around an axis can significantly exceed $2 \pi$. The more the number of electron revolutions, the greater the time the electron stays in the diode gap, and there should be less emission current from the cathode surface. Thus, the steady state of an electron flow depends on the value of emission current chosen (and an electric field on the cathode surface equal to zero corresponding to a space charge limited current; but the value of emitted current is much less than limiting current and, basically, can approach zero).

\section{PARAPOTENTIAL (BRILLOUIN) MODEL[1]}

A system of units in which $c=e=m_{e}=1$ is hereafter used. The electronic flow consists of circular trajectories with radii $r$ filling completely or partially the gap between the cathode with radius $r_{k}$ and anode with radius $r_{a}: r_{k}<$ $r<r_{e}, r_{e} \leq r_{a}$.

Outer (boundary) parameters - anode voltage $\left(\gamma_{a}-1\right)$ and (kept in a short pulse regime) average value of magnetic induction in the diode gap $B_{0}-$ are connected to parameters of an electronic flow by the relations

$$
\begin{gathered}
\ln \left(\frac{r_{e}}{r_{k}}\right)=\int_{0}^{A_{e}} \frac{d x}{\sqrt{\left(C^{2}+x^{2}\right)\left(1+x^{2}\right)}}, \\
\gamma_{a}=\gamma_{e}+r_{e} E_{e} \ln \left(\frac{r_{a}}{r_{e}}\right), \\
r_{a} A_{a}=\frac{r_{a}^{2}-r_{k}^{2}}{2} B_{0}=r_{e} A_{e}+\frac{r_{a}^{2}-r_{e}^{2}}{2} B_{e}
\end{gathered}
$$


from which in that specific case $r_{e}=r_{a}$ (electronic flow occupies the whole anode-cathode space) is essentially only the last relation between an average magnetic induction and diode voltage:

$$
\left(r_{a}^{2}-r_{k}^{2}\right) B_{0}=2 r_{a} \sqrt{\gamma_{a}^{2}-1} .
$$

\section{KINETIC (EMISSIONING) MODEL[4].}

This analytical model, supposing presence of an emission current from the cathode coming back on the cathode, is described by a system of equations for $\gamma(r), A(r)$

$$
\begin{gathered}
\frac{1}{r} \frac{d}{d r}\left(r \frac{d \gamma}{d r}\right)=\frac{f_{0}}{r} \frac{\gamma}{\sqrt{\gamma^{2}-1-A^{2}}}, \\
\frac{d}{d r}\left(\frac{1}{r} \frac{d}{d r}(r A)\right)=\frac{f_{0}}{r} \frac{A}{\sqrt{\gamma^{2}-1-A^{2}}}, \\
\frac{d \gamma}{d r}=E, \quad \frac{1}{r} \frac{d}{d r}(r A)=B,
\end{gathered}
$$

where the constant $f_{0}$ is proportional to density of cathode emission current. On the cathode $\gamma\left(r_{k}\right)=1, A\left(r_{k}\right)=0$, $E\left(r_{k}\right)=0, B\left(r_{k}\right)=B_{k}$, and the outer boundary of electron flow is at radius $r_{e}$, at which electrons turn back to the cathode and the radial pulse of electrons $p_{r}=$ $\sqrt{\gamma^{2}-1-A^{2}}$ is equal to zero. In this model anode voltage and average value of magnetic induction are connected to parameters of an electronic flow by the relations:

$$
\begin{gathered}
\gamma_{a}=\gamma_{e}+r_{e} E_{e} \ln \left(\frac{r_{a}}{r_{e}}\right), \\
r_{a} A_{a}=\frac{r_{a}^{2}-r_{k}^{2}}{2} B_{0}=r_{e} A_{e}+\frac{r_{a}^{2}-r_{e}^{2}}{2} B_{e}
\end{gathered}
$$

The results of calculations on these models are shown in Fig. 1 for the same external conditions (voltage and external magnetic flux) and with zero electric field on the cathode. The Brillouin smooth solution and three kinetic ones from a set of possible number of layers $(\mathrm{n}=1,2,4)$ are shown. With increasing number of layers, emission current from the cathode tends to zero, electrons make more and more revolutions before coming back to the cathode, and a kinetic solution gradually approaches the Brillouin one. In the situation when magnetic field is much stronger than electric field, the Brillouin solution appears to be the only possible one. Thus the kinetic solution occurs only if the electric field on the cathode is not equal to zero. We note that in the inverted magnetron diode (with the anode on inner surface) only a single-layer kinetic solution exists, which points to the essential influence of cylindricity on electron flow within the diode. In "plane" approximation for the kinetic model the criterion for restriction of number of layers by any value is not present, and the usual choice of $\mathrm{n}=1$ is in essence arbitrary.
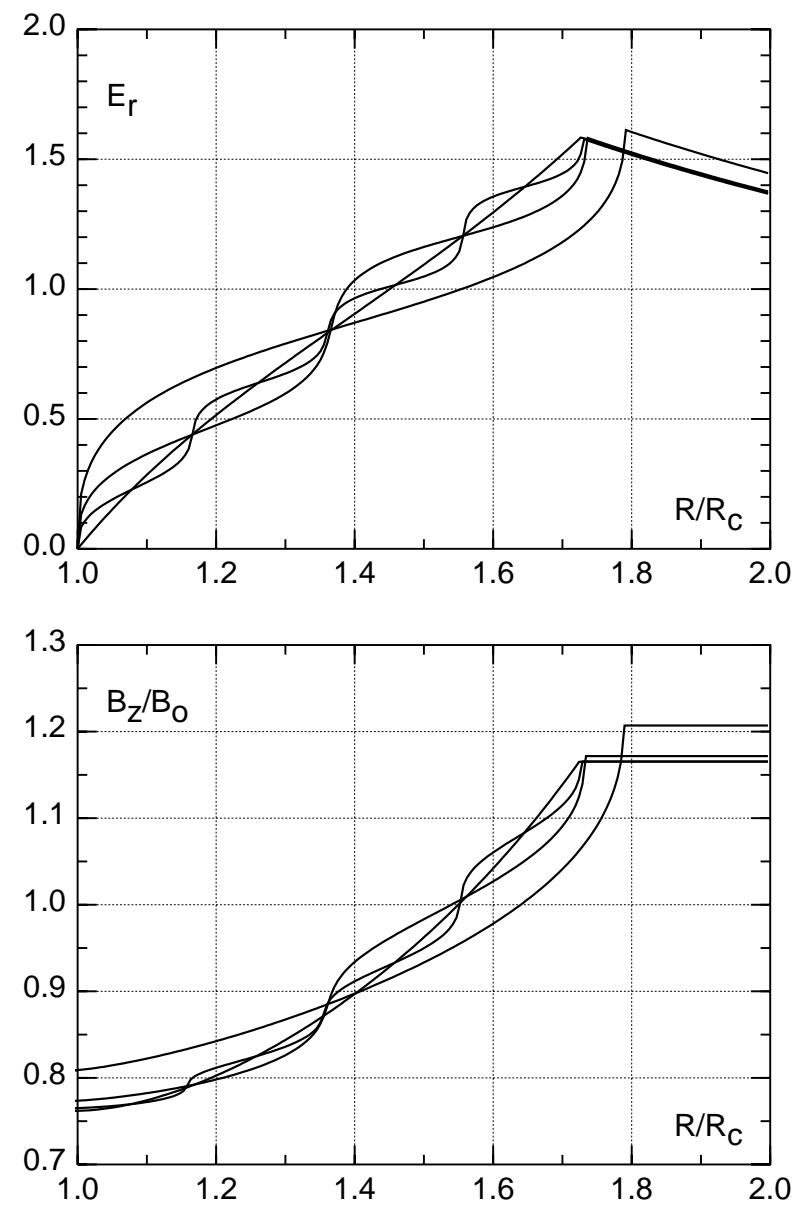

Figure 1: Kinetic $(n=1,2,4)$ and Brillouin models of a rotating beam $(\mathrm{E}(\mathrm{r})$ upper, $\mathrm{B}(\mathrm{r})$ lower) for the same external conditions (voltage and magnetic flux).

\section{TEMPORAL EFFECTS AND THEORETICAL MODELS}

Discussion of effects observed in a magnetron diode had as its basic purpose to show that theoretical models describing, one should think, one and the same situation actually correspond to various physical conditions. We shall illustrate this by an example, comparing analytical results and numeric simulation realized with a PIC-code KARAT[6].

Modeling of particle emission in KARAT code can be realized in two ways: (1) by setting the law for temporal change of emitted current, the value of which can be less than, or more than limiting current, the applied voltage being fixed (this situation corresponds to emission from photocathodes or external injection of a beam through the surface of the emitter); and (2) by setting the law for temporal rising of diode voltage to some constant value, emission current being fixed at a value greatly exceeding the value limited by space charge (this situation corresponds to thermionic cathode). In Fig. 2 typical distribution functions of particles in these two cases are shown. In the first case a single electron revolution in a cycloid is realized (symmetric two-peak distribution of electrons on a pulse $p_{r}$ ) 

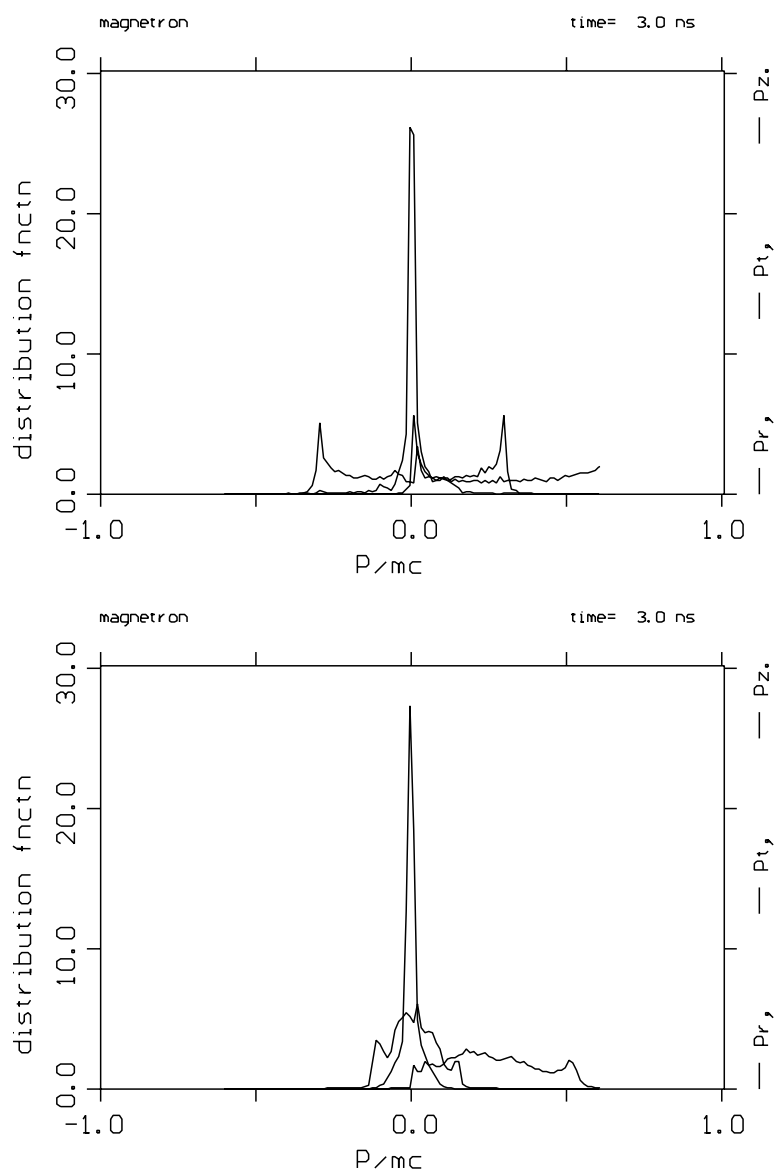

Figure 2: Example of distribution functions.

without accumulation of charge in an accelerating gap; in the second case - symmetric distribution of particles on a pulse $p_{r}$ with capture of electrons during voltage increase, growth of number of particles in diode gap and multiturn dynamics of electrons.

\section{DISCUSSION}

Both theoretical models give about the same physical results. Recall that the equations describing Brillouin flow can be deduced on the basis of the same approach used for the kinetic model [7]. Under conditions of conservation of full energy of particles and canonical angular momentum

$$
P_{\theta}=r\left(p_{\theta}+A\right)=\text { const }
$$

the next general set of equations can be derived:

$$
\begin{gathered}
\left(r \gamma^{2} v_{z}^{\prime}\right)^{\prime}+\gamma\left(\frac{v_{\theta} P_{\theta}^{\prime}}{v_{z}}\right)^{\prime}=0, \\
\left(r \gamma^{2} v_{\theta}^{\prime}\right)^{\prime}-\frac{\gamma^{2} v_{\theta}}{r}-r \gamma\left(\frac{P_{\theta}^{\prime}}{r}\right)^{\prime}=0,
\end{gathered}
$$

where the prime denotes $d / d r$.

For the case of a magnetron diode with a cathode surface coinciding with a magnetic flux surface we have $P_{\theta}=$ const and

$$
r \gamma^{2} v_{z}^{\prime}=\text { const. }
$$

The constant equals zero (i.e., $v_{z}=$ const) in this case and we have the same equations as in Section III [8].

Therefore, it is not surprising that a direct transition exists from kinetic flow to a Brillouin one as a limit.

Work supported by RFFI under grant 96-02-19215a.

\section{REFERENCES}

[1] A.V. Agafonov, V.S. Voronin, A.N. Lebedev, K.N. Pazin. JTP, 1974, v. 44, p. 1909.

[2] J.M. Creedon. J. Appl. Phys., 1977, v. 48, p.1070.

[3] R.V. Lovelace, E. Ott. Phys. Fluids, 1974, v. 17, p. 1263.

[4] V.S. Voronin, A.N. Lebedev. JTP, 1973, v. 43, p. 2591.

[5] A.V. Agafonov, D.B. Orlov. Proc. of the 1989 IEEE Particle Accelerator Conference, Chicago, USA, v. 2, p. 1397.

[6] P.V. Kotetashwily, P.V. Rybak, V.P. Tarakanov, Inst. of Gen. Phys., Moscow, Russia, prepr. no. 44, 1991.

[7] M. Raiser. Phys. Fluids 1974, v. 20, p. 477.

[8] A.V. Agafonov, Proc. of the 1995 IEEE Particle Accelerator Conference, Dallas, USA, v. 5, p. 3272. 